\title{
Light at night acutely impairs glucose tolerance in a time-, intensity- and wavelength-dependent manner in rats
}

\author{
Anne-Loes Opperhuizen ${ }^{1,2}$ • Dirk J. Stenvers ${ }^{2,3}$ • Remi D. Jansen ${ }^{1}$ • Ewout Foppen ${ }^{2,3}$ • \\ Eric Fliers ${ }^{3} \cdot$ Andries Kalsbeek $^{1,2,3}$
}

Received: 12 December 2016 / Accepted: 2 March 2017/Published online: 3 April 2017

(C) The Author(s) 2017. This article is published with open access at Springerlink.com

\begin{abstract}
Aims/hypothesis Exposure to light at night (LAN) has increased dramatically in recent decades. Animal studies have shown that chronic dim LAN induced obesity and glucose intolerance. Furthermore, several studies in humans have demonstrated that chronic exposure to artificial LAN may have adverse health effects with an increased risk of metabolic disorders, including type 2 diabetes. It is well-known that acute exposure to LAN affects biological clock function, hormone secretion and the activity of the autonomic nervous system, but data on the effects of LAN on glucose homeostasis are lacking. This study aimed to investigate the acute effects of LAN on glucose metabolism.

Methods Male Wistar rats were subjected to i.v. glucose or insulin tolerance tests while exposed to $2 \mathrm{~h}$ of LAN in the early or late dark phase. In subsequent experiments, different light intensities and wavelengths were used.

Results LAN exposure early in the dark phase at ZT15 caused increased glucose responses during the first $20 \mathrm{~min}$ after glucose infusion $(p<0.001)$, whereas LAN exposure at the end of the dark phase, at ZT21, caused increased insulin responses during the first $10 \mathrm{~min}(p<0.01)$, indicating that LAN
\end{abstract}

Anne-Loes Opperhuizen

a.opperhuizen@nin.knaw.nl

1 Hypothalamic Integration Mechanisms, Netherlands Institute for Neuroscience (NIN), Meibergdreef 47, 1105 BA Amsterdam, the Netherlands

2 Laboratory of Endocrinology, Department of Clinical Chemistry, Academic Medical Centre (AMC) University of Amsterdam, Amsterdam, the Netherlands

3 Department of Endocrinology and Metabolism, Academic Medical Center (AMC) University of Amsterdam, Amsterdam, the Netherlands immediately induces glucose intolerance in rats. Subsequent experiments demonstrated that the effect of LAN was both intensity- and wavelength-dependent. White light of 50 and $150 \mathrm{~lx}$ induced greater glucose responses than 5 and $20 \mathrm{~lx}$, whereas all intensities other than $5 \mathrm{~lx}$ reduced locomotor activity. Green light induced glucose intolerance, but red and blue light did not, suggesting the involvement of a specific retina-brain pathway.

Conclusions/interpretation Together, these data show that exposure to LAN has acute adverse effects on glucose metabolism in a time-, intensity- and wavelength-dependent manner.

Keywords Biological clock · Glucose intolerance · Green light $\cdot$ Insulin sensitivity $\cdot$ Light at night $\cdot$ Rat $\cdot$ Rodent

\author{
Abbreviations \\ ANS Autonomic nervous system \\ ipRGC Intrinsically photosensitive retinal ganglion cell \\ IVITT Intravenous insulin tolerance test \\ LAN Light at night \\ LEDs Light-emitting diodes \\ SCN Suprachiasmatic nucleus \\ ZT Zeitgeber time
}

\section{Introduction}

Electric lighting is widely used around the world, but possible harmful effects of the presence of light $24 \mathrm{~h}$ a day 7 days a week have become apparent only recently. In particular, the use of light at night (LAN) is considered potentially disruptive, as organisms are not physiologically prepared to deal with light signals during the night. Light is caught by the eyes and transformed into electrical signals by retinal receptors and 
conducted through the optic nerves to the brain areas important for vision, autonomic functions and circadian timing. Historically, sunlight would be mainly responsible for these functions, but artificial light with the appropriate wavelength and intensity may mimic the effects of sunlight.

Exposure to LAN has been correlated with an increased risk of developing obesity [1,2], diabetes [3] and dyslipidaemia [2] in humans. In addition, rodent studies showed that chronic exposure to LAN reduces the amplitude of the sleep/wake rhythm [4-6], increases body weight and decreases glucose tolerance $[4,5]$. Acute exposure to LAN has been shown to alter the profiles of the hormones melatonin and corticosterone [7, 8]. Moreover, LAN alters clock gene expression in the hypothalamic circadian clock [9], and affects gene expression in peripheral organs [7, 10]. Light is the most important cue for the circadian clock to synchronise its endogenously generated rhythmic activity with the environmental light/dark cycle. The clock, located in the suprachiasmatic nucleus ( $\mathrm{SCN}$ ), transmits timing information to downstream targets via hormones and the autonomic nervous system (ANS) to optimally prepare the organism for regular daily changes. For example, the SCN directly controls glucose homeostasis, including basal glucose levels and glucose tolerance [11, 12], at least partly through the ANS [13].

Visual and non-visual effects of light are mediated initially by retinal cells. Intense research into the non-visual effects of light revealed the existence of a novel retinal photoreceptor, the intrinsically photosensitive retinal ganglion cells (ipRGCs), which express the photopigment melanopsin [14, 15]. Knowledge of rods and cones is well established, and these cells are still considered to be the most important photoreceptors for vision, with rods being dominantly active in dim light and cones in bright light conditions. The ipRGCs provide the majority of retinal input to non-visual brain structures and dominantly innervate the SCN, providing information essential for circadian physiology [15]. However, the visual and non-visual pathways are not completely separate entities, as ipRGCs also receive indirect input from rods and cones [15-17].

The correlation between LAN and metabolic disorders together with the power of light to control the SCN and downstream targets led to the development of a hypothesis that LAN affects glucose homeostasis. The current study tested this hypothesis by subjecting Wistar rats to glucose and insulin tolerance tests during acute LAN at the beginning and end of the dark phase. As a secondary objective, we investigated glucose intolerance induced by acute LAN exposure in the early dark phase as a function of light intensity and wavelength.

\section{Materials and methods}

Animals and housing Male Wistar rats (Charles River Breeding Laboratories, Sulzfeld, Germany) weighing 300-
$350 \mathrm{~g}$ were maintained under a controlled 12/12 light/dark cycle (lights on 07:00 hours, Zeitgeber Time 0 (ZT0); lights off 19:00 hours, ZT12), with ad libitum access to water and a regular chow diet (Teklad Global Diet, Harlan, Horst, the Netherlands), unless stated otherwise. Red dim light (max. 5 photopic lux [lx]) was present in the room during the dark phase, whereas mixed white fluorescent light (max. $150 \mathrm{~lx}$ ) was present during the light phase. All experimental procedures were performed in accordance with the Council Directive 2010/63EU of the European Parliament and the Council of 22 September 2010 on protection of animals used for scientific purposes. All experimental procedures were approved by the Animal Ethics Committee of the Royal Dutch Academy of Arts and Sciences (KNAW, Amsterdam, the Netherlands) and in accordance with the guidelines on animal experimentation of the Netherlands Institute for Neuroscience.

Experimental design After adaptation to the animal facility, all rats, except the experimental groups for locomotor activity recordings, were anaesthetised with $80 \mathrm{mg} / \mathrm{kg}$ ketamine (Eurovet Animal Health, Bladel, the Netherlands), $8 \mathrm{mg} / \mathrm{kg}$ Rompun (xylaxine, Bayer Health Care, Mijdrecht, the Netherlands) and $0.1 \mathrm{mg} / \mathrm{kg}$ atropine (Pharmachemi, Haarlem, the Netherlands). Subsequently, an intra-atrial silicone catheter was implanted unilaterally in the jugular vein and fixed on the skull [12]. Animals were left to recover for at least 1 week before the start of experiments. To study whether LAN affects glucose or insulin tolerance, animals were exposed to $2 \mathrm{~h}$ of light during which an IVGTT or intravenous insulin tolerance test (IVITT) [12] was performed. Food was removed $2.5 \mathrm{~h}$ prior to the tests. In a randomly assigned crossover design, all animals included in the final data sets were tested twice, once under control (i.e. dim red light) and once under experimental (i.e. LAN) conditions, with a 1 week recovery period between. Animals with incomplete recovery of body weight or blockage of the catheter were excluded from the experiment.

Experiment 1 Animals were randomly assigned to one of two groups to study the effects of LAN (white light $\sim 125 \pm 25 \mathrm{~lx}$ ) on glucose tolerance: group 1A $(n=11)$ where under LAN conditions the light was turned on from ZT14-16 and the IVGTT started at ZT15, and under control conditions the IVGTT started at ZT15; and group $1 \mathrm{~B}(n=10)$ where under LAN conditions the light was turned on from ZT20-22 and the IVGTT started at ZT21, and under control conditions the IVGTT started at ZT21.

Two additional groups of animals were used to study insulin tolerance using an IVITT under the same light conditions as described for groups 1A and 1B. Group 1C $(n=13)$ was exposed to $2 \mathrm{~h}$ LAN from ZT14-16 and IVITT started at ZT15, and group 1D $(n=7)$ was exposed to $2 \mathrm{~h}$ LAN from ZT20-22 and IVITT started at ZT21. 
Experiment 2 Four groups were included to study the effect of different light intensities. The conditions were similar to experiment 1A: mixed white LAN from ZT14-16 and an IVGTT started at ZT15. Animals were randomly assigned to one of four groups: $5 \mathrm{~lx}(n=10), 20 \mathrm{~lx}(n=7), 50 \mathrm{~lx}(n=9)$ or $150 \mathrm{~lx}(n=9)$.

To study the effects of LAN on locomotor activity, four additional groups of animals were solitary housed in transparent cages to record locomotor activity with pressure plates [12]. Each measurement included $48 \mathrm{~h}$ continuous recording with 6 min intervals. In the first $24 \mathrm{~h}$, locomotion was measured while animals were exposed to the normal (12/12) lighting schedule. In the second $24 \mathrm{~h}$ recording period, animals were exposed to LAN from ZT14-16. LAN exposure during this $2 \mathrm{~h}$ period was at $5 \mathrm{~lx}(n=7), 20 \mathrm{~lx}(n=5), 50 \mathrm{~lx}(n=6)$ or $150 \mathrm{~lx}(n=6)$.

Experiment 3 Four groups were included to study the effects of wavelength. The conditions were similar to experiment $1 \mathrm{~A}$. Animals were randomly assigned to one of four groups and exposed to white light ranging from $400-700 \mathrm{~nm}(n=9)$, blue light with a peak at $457 \mathrm{~nm}(n=9)$, green light with a peak at $520 \mathrm{~nm}(n=8)$ or red light with a peak at $633 \mathrm{~nm}(n=10)$.

Four additional groups of animals were included as described in experiment 2 to study the effects of wavelength on locomotor activity. LAN exposure was the same as for the IVGTTs: white $(n=8)$, blue $(n=9)$, green $(n=9)$ or red $(n=9)$.

Light exposure White light exposure during experiments 1 and 2 was done with SMD3528 light-emitting diodes (LEDs; Watshome). Light exposure of different wavelengths in experiment 3 was done with SMD5050 LEDs (Watshome). Adhesive strips of LEDs were attached to in-house-built plastic walls surrounding the animal cages with horizontal light exposure at eye level of the animals. LEDs were controlled by software built in-house. The illuminance of the light for the different conditions in experiment 3 was adjusted such that photon flux and irradiance were similar between LAN conditions (see Table 1 for irradiance spectrum [18]).

Plasma measurements Blood samples were cooled after collection, subsequently centrifuged $\left(4^{\circ} \mathrm{C}, 1699 \mathrm{~g}, 15 \mathrm{~min}\right)$ and plasma was stored at $-20^{\circ} \mathrm{C}$ until further analysis. Plasma glucose concentrations were determined immediately after blood sampling with a blood glucose monitoring system (FreeStyle Freedom-Lite, Abbott Diabetes Care, Alameda, CA, USA). Radioimmunoassays were used according to the manufacturer's protocol to measure plasma insulin concentrations (Millipore, St Charles, MO, USA) and plasma corticosterone concentrations (MP Biomedicals, Santa Ana, CA, USA). Samples with incomplete duplicates due to technical reasons were excluded from the data.

Statistical analysis All data are expressed as means \pm SEM. Paired $t$ tests were used to detect group differences in baseline concentrations for glucose (experiments 1, 2 and 3), insulin (experiments 1 and 3 ) and corticosterone (experiments 1 and 3 ). Paired $t$ tests were also used to detect group differences in locomotor activity in experiments 2 and 3 . A repeated measures two-way ANOVA was used to test for effects of treatment (control or LAN), time or interaction (treatment $\times$ time) on the responses of glucose (experiments 1,2 and 3), insulin (experiments 1 and 3 ) and corticosterone (experiments 1 and 3 ). If a treatment or interaction effect was found, post hoc Sidaks multiple comparisons tests were used to determine differences between light conditions at individual time points. The net AUC was determined from 0-60 min using the trapezoid rule. Delta values are calculated by subtracting the baseline $(t=0 \mathrm{~min})$ from each time sample $(t=5,10,20,30$ and $60 \mathrm{~min}$ ) raw data value. Paired $t$ tests were used to detect group differences in AUC for glucose (experiments 1, 2 and 3 ), insulin (experiments 1 and 3 ) and corticosterone (experiments 1 and 3). All statistical analyses were performed with GraphPad Prism version 7.01 for Windows (GraphPad Software, La Jolla, CA, USA) using a significance level of $p<0.05$.
Table 1 Spectral sensitivity of light conditions used in experiment 3

\begin{tabular}{lllll}
\hline Retinal photopigment complement & White & Blue & Green & Red \\
\hline S-cone $\left(\mathrm{rN}_{\mathrm{sc}}[\lambda]\right)$ & 0.41 & 1.46 & 0.17 & 0.08 \\
Melanopsin $\left(\mathrm{rN}_{\mathrm{z}}[\lambda]\right)$ & 52.61 & 265.07 & 150.85 & 1.40 \\
Rod $\left(\mathrm{rN}_{\mathrm{r}}[\lambda]\right)$ & 64.08 & 202.74 & 212.35 & 1.50 \\
M-cone $\left(\mathrm{rN} \mathrm{mc}_{\mathrm{m}}[\lambda]\right)$ & 72.84 & 168.80 & 232.98 & 2.10 \\
Irradiance $\left(\mu \mathrm{W} / \mathrm{cm}^{2}\right)$ & 46.01 & 52.95 & 47.24 & 23.23 \\
Photon flux $\left(1 \mathrm{~cm}^{-2} \mathrm{~s}^{-1}\right)$ & $1.35 \times 10^{14}$ & $1.28 \times 10^{14}$ & $1.28 \times 10^{14}$ & $0.74 \times 10^{14}$ \\
\hline
\end{tabular}

All data based on the rodent-toolbox provided by Lucas et al [18]

The upper rows represent weighted contribution of rodent retinal photopigments (S-cone, ipRGC [melanopsin], rod, M-cone) in $\alpha$-opic rodent lux. The lower rows represent unweighted characteristics of the LEDs (irradiance, photon flux) 


\section{Results}

Experiment 1 Effects of acute exposure to LAN on glucose homeostasis were tested by exposing rats to $2 \mathrm{~h}$ light at the beginning (ZT14-16) or end (ZT20-22) of the dark phase. In the early dark phase (ZT15), LAN induced increased plasma glucose levels after glucose infusion (Fig. 1a, b). No significant differences were observed for insulin (Fig. 1e, f). In the late dark phase (ZT21), LAN caused a trend towards increased plasma glucose (Fig. 1c, d). In contrast to ZT15, insulin plasma levels were increased by LAN at ZT21 after glucose infusion (Fig. 1g), resulting in a trend towards a higher AUC for insulin (Fig. 1h). Corticosterone responses were unaffected by LAN at ZT15 or ZT21 (Table 2).
Tolerance tests were initiated at the start of the second hour of the light exposure; therefore, baseline samples $(t=0 \mathrm{~min})$ were obtained after $1 \mathrm{~h}$ light exposure (both ZT15 and ZT21). Baseline glucose concentrations were unaffected by $1 \mathrm{~h} \mathrm{LAN}$ at both time points (Fig. 2a). Baseline insulin concentrations tended to be lower both at ZT15 and ZT21 (Fig. 2b). Baseline levels of corticosterone were slightly reduced by LAN at ZT15 and showed a trend towards decreased levels at ZT21 (Fig. 2c).

To investigate whether LAN affects insulin sensitivity, animals were exposed to an IVITT. LAN did not induce changes in plasma glucose concentrations after insulin infusion at ZT15 (Fig. 3a, b) or ZT21 (Fig. 3d, e). Also corticosterone responses were not different between control and LAN conditions. Baseline concentrations of glucose (ZT15 Fig. 3c,
Fig. 1 LAN decreases glucose tolerance at ZT15 and increases insulin responses at ZT21. LAN from ZT14-16 decreased glucose tolerance with higher glucose concentrations $(\mathbf{a}, \mathbf{b})$ and unchanged insulin responses (e, f). LAN from ZT20-22 did not affect plasma glucose concentrations (AUC $p=0.08$ ) (c, d), but increased insulin concentrations (AUC $p=0.059$ ) $(\mathbf{g}, \mathbf{h})$. AUC figures show the group mean on the left and individual animals on the right. Control (C), solid lines, black columns and symbols; LAN, dashed lines, white columns and symbols. $* p<0.05, * * p<0.01$, $* * * p<0.001$ a

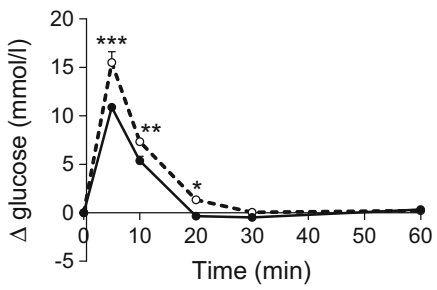

C
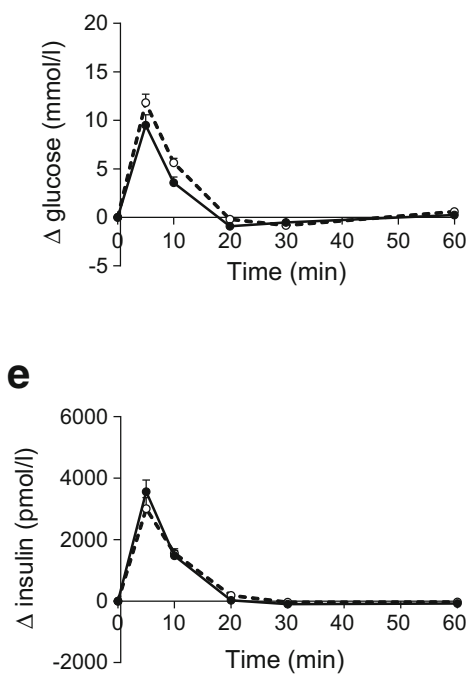

g

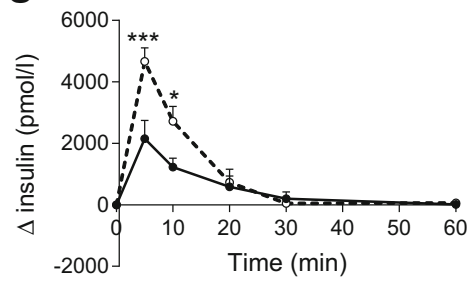

b
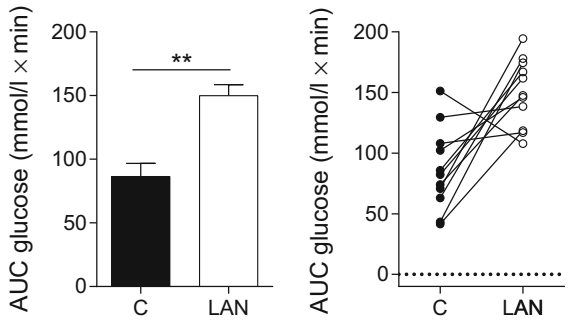

d
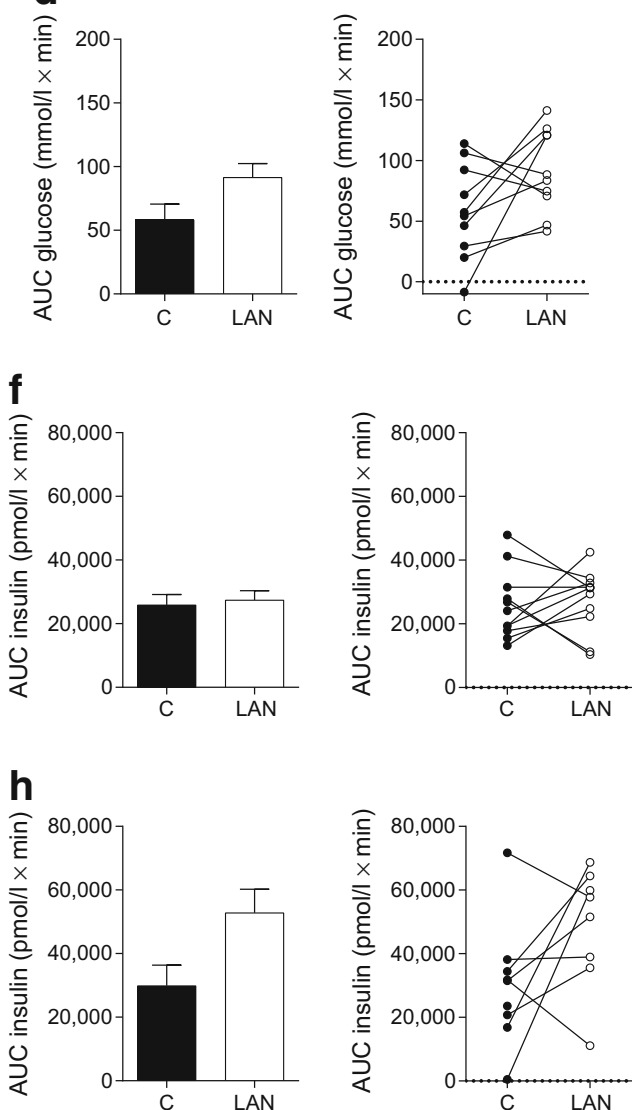
Table 2 Statistical analyses of effects of light on glucose, insulin and corticosterone responses during glucose and insulin tolerance tests in experiment 1

\begin{tabular}{cllccc}
\hline Experiment & Variable & $p$ for treatment & $p$ for time & $p$ for interaction & $p$ for AUC \\
\hline IVGTT & & & & & \\
ZT15 & Glucose & 0.002 & $<0.001$ & $<0.001$ & 0.003 \\
& Insulin & 0.769 & $<0.001$ & 0.122 & 0.719 \\
& Corticosterone & 0.208 & 0.560 & 0.518 & 0.254 \\
\multirow{2}{*}{ ZT21 } & Glucose & 0.090 & $<0.001$ & 0.063 & 0.080 \\
& Insulin & 0.015 & $<0.001$ & 0.004 & 0.058 \\
& Corticosterone & 0.648 & 0.054 & 0.585 & 0.732 \\
IVITT & & & & \\
ZT15 & Glucose & 0.106 & $<0.001$ & 0.298 & 0.118 \\
& Corticosterone & 0.532 & $<0.001$ & 0.312 & 0.975 \\
ZT21 & Glucose & 0.817 & $<0.001$ & 0.250 & 0.617 \\
& Corticosterone & 0.061 & 0.114 & 0.240 & 0.069 \\
\hline
\end{tabular}

Treatment (light condition), time (sample time) and interaction effects were determined using repeated measures two-way ANOVA

Glucose, insulin and corticosterone responses are reported for the IVGTT at ZT15 and ZT21. Glucose and corticosterone responses are reported for the IVITT at ZT15 and ZT21

The AUC column contains results of a paired $t$ test on a net AUC curve
ZT21 Fig. 3f) and corticosterone (ZT15: $41 \pm 9$ in control vs $70 \pm 19$ in LAN, $p=0.232$; ZT21: $41 \pm 16$ vs $61 \pm 27$, $p=0.388$ ) were unaffected by LAN at both time points. The statistical analyses of experiment 1 are reported in Table 2.

Experiment 2 The effects of LAN on glucose tolerance were largest in the early dark phase (at ZT15) and therefore this time point was chosen to further investigate the characteristics of light-induced glucose intolerance. Four different light intensities were used to test whether the effects of light on glucose tolerance depend on intensity. Significantly higher peaks were found after glucose infusions in 50 and $150 \mathrm{~lx}$ conditions when compared with 5 and $201 x$ (time $p<0.001$; treatment [intensity] $p=0.013$; interaction [intensity $\times$ time] $p=0.007)$. A significant treatment effect was only observed in the 50 and $150 \mathrm{~lx}$ conditions with higher glucose levels in LAN conditions when compared with control (Fig. 4a-d). AUC was significantly higher with $50 \mathrm{~lx}$ and tended to be at $150 \mathrm{~lx}$. Baseline levels of glucose were unaffected by 5, 20 or $150 \mathrm{~lx}$, and slightly reduced with 50 lx LAN (Fig. 4e-h). Locomotor recordings during LAN and control conditions showed decreased locomotion with 20 , 50 and $150 \mathrm{~lx}$. The decrease was significant for 20 and $150 \mathrm{~lx}$ (20 lx $p=0.001 ; 150 \mathrm{~lx} p=0.007$ ), with a similar trend for $50 \mathrm{~lx}(p=0.052)$. Locomotor activity in animals exposed to 5 lx was unaffected ( $p=0.915$, Fig. $4 \mathrm{i}-1)$. The statistical analyses of experiment 2 are reported in Table 3.

Experiment 3 To study whether LAN-induced glucose intolerance was wavelength-dependent, four different wavelengths were used: short (blue), middle (green) and long (red), as well as broad-spectrum white light including all wavelengths (Table 1). As reported for experiments 1 and 2, white LAN induced glucose intolerance during a $2 \mathrm{~h}$ exposure starting at ZT14 (Fig. 5a, b). Increased glucose concentrations in the LAN condition were also observed in green light (Fig. 5i, j), but not in blue (Fig. 5e, f) or red light (Fig. 5m, n). Insulin responses were not affected by wavelength (Fig. $5 \mathrm{c}-\mathrm{o}$ ). Corticosterone responses were unaffected by blue, green or red light, but were
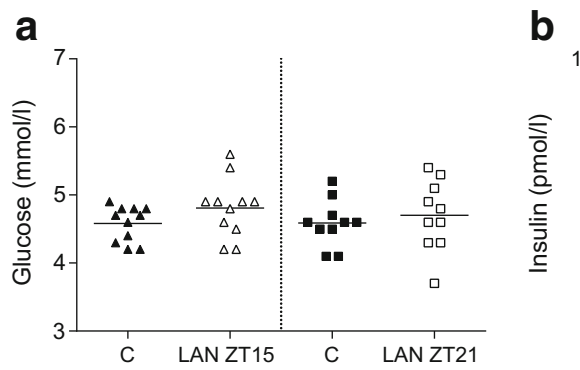

Fig. 2 LAN decreases baseline corticosterone at ZT15 but does not affect baseline glucose and insulin concentrations. No significant differences were observed in baseline glucose (a) and insulin concentrations (b)

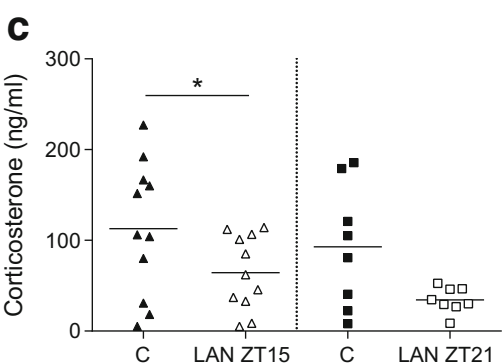

between control (black symbols) and LAN (white symbols) conditions during experiment 1 . LAN significantly decreased basal corticosterone concentrations at ZT15, but not at ZT21 (c). $* p<0.05$ 
Fig. 3 Blood glucose concentrations are unaffected by LAN during IVITT at ZT15 and ZT21. LAN did not alter the decrease in blood glucose after insulin infusion at ZT15 (a, b) or ZT21 (d, e). Baseline

concentrations were not affected by LAN at ZT15 (c) or ZT21 (f) when compared with control conditions. Control (C), solid lines, black columns and symbols; LAN, dashed lines, white columns and symbols a

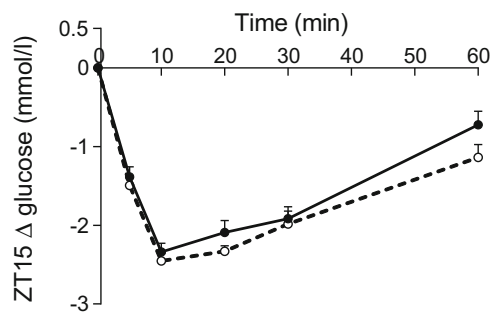

d

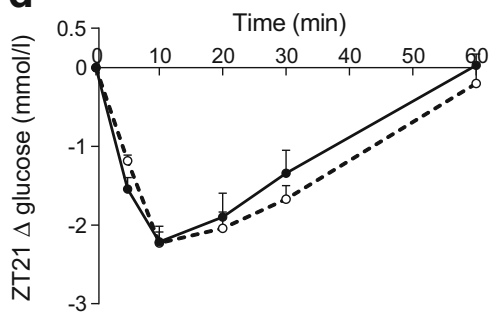

b
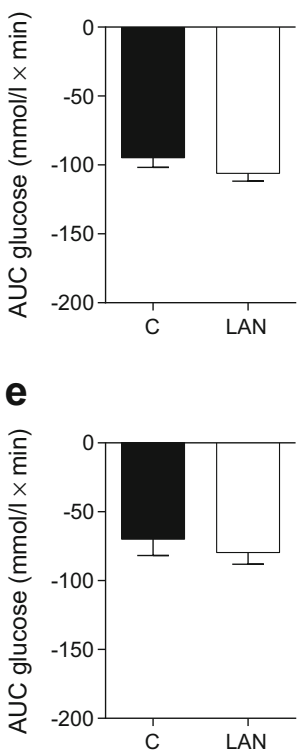

C

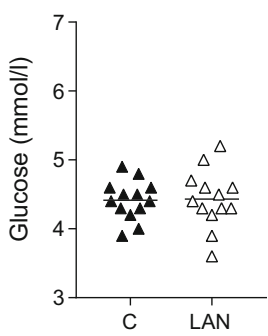

f

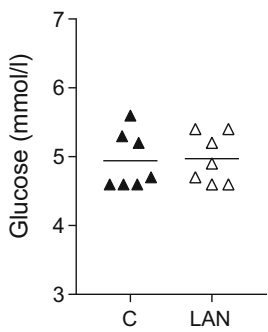

significantly increased by white light exposure. Locomotor activity recordings demonstrated that white $(p=0.026)$, blue $(p=0.019)$ and green $(p=0.028)$, but not red $(p=0.812)$, light decreased locomotion compared with the control dark
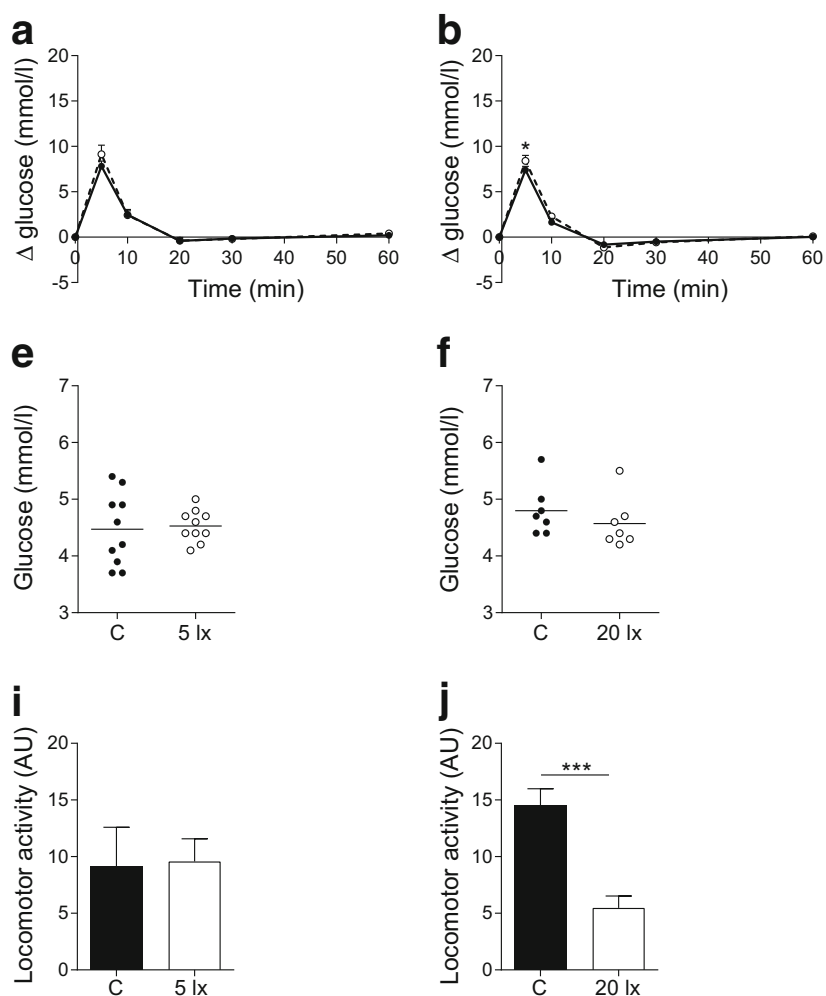

Fig. 4 The effects of LAN on glucose tolerance and locomotor activity are intensity-dependent. LAN with intensity of $5 \mathrm{~lx}$ (a) did not induce changes in blood glucose concentrations, but $20 \mathrm{~lx}(\mathbf{b}), 50 \mathrm{~lx}$ (c) and $150 \mathrm{~lx}$ (d) did. Baseline glucose levels were unaffected in $5 \mathrm{~lx}(\mathbf{e}), 20 \mathrm{~lx}$ (f) or $150 \mathrm{~lx}$ (h) conditions, but slightly decreased by LAN of $50 \mathrm{~lx}(\mathrm{~g})$. conditions (Fig. 5d-p). Baseline concentrations of glucose, insulin and corticosterone were unaffected by any of the light conditions (Fig. 6). The statistical analyses of experiment 3 are reported in Table 4.
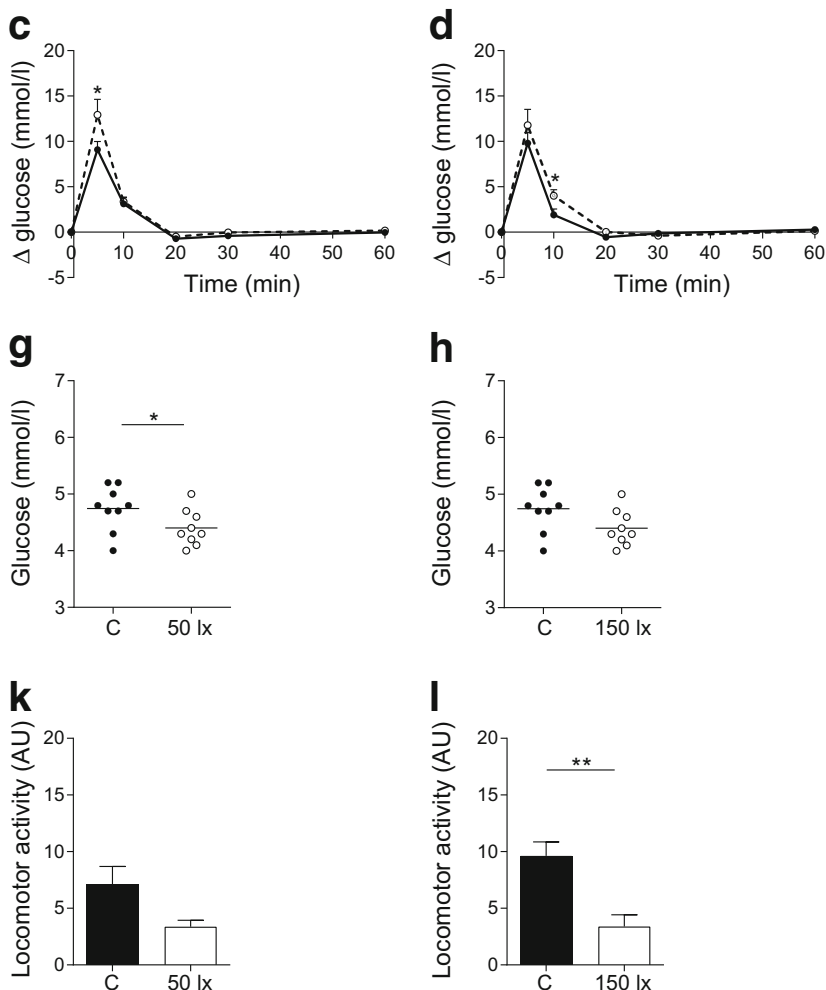

Total locomotor activity levels recorded from ZT14-16 were reduced during light conditions compared with control conditions for $20 \mathrm{~lx} \mathrm{(j)}$, $501 \mathrm{x}(p=0.05)(\mathbf{k})$ and $150 \mathrm{~lx}(\mathbf{I})$, but not $5 \mathrm{~lx}$ (i). Control (C), solid lines, black columns and symbols; LAN, dashed lines, white columns and symbols. $* p<0.05, * * p<0.01, * * * p<0.001$ 
Table 3 Statistical analyses of effects of light on glucose responses during glucose tolerance tests in experiment 2

\begin{tabular}{llllcc}
\hline Intensity & Variable & $p$ for treatment & $p$ for time & $p$ for interaction & $p$ for AUC \\
\hline $5 \mathrm{~lx}$ & Glucose & 0.383 & $<0.001$ & 0.078 & 0.524 \\
$20 \mathrm{~lx}$ & Glucose & 0.362 & $<0.001$ & 0.015 & 0.686 \\
$50 \mathrm{~lx}$ & Glucose & 0.010 & $<0.001$ & $<0.001$ & 0.009 \\
$150 \mathrm{~lx}$ & Glucose & 0.030 & $<0.001$ & 0.073 & 0.106 \\
\hline
\end{tabular}

Treatment (intensity), time (sample time) and interaction effects were determined using repeated measures twoway ANOVA

Glucose responses are reported for the IVGTT during four light intensities

The AUC column contains results of paired $t$ test on the net AUC curve. The threshold for significance is $p<0.05$

\section{Discussion}

We show that exposure to LAN causes acute glucose intolerance in rats and that this effect is dependent on the time of day, intensity and wavelength of the light exposure. The LANinduced glucose intolerance at the start of the dark period was reflected by increased plasma glucose levels, whereas LAN-induced glucose intolerance at the end of the dark period was mainly reflected by increased plasma insulin. Surprisingly, green, but not blue, light best mimicked the effects of white light. These results suggest an important role for middle-wavelength cones in the LAN-induced effects on glucose intolerance.

Most non-visual light responses, such as the pupillary light response, melatonin inhibition and modulation of heart rate, are exerted via the ANS. Animal studies have shown that LAN acutely increases sympathetic activity and decreases parasympathetic activity of the autonomic nerves innervating peripheral organs, including the liver and pancreas [7, 19, 20]. Moreover, denervation of target organs, such as the liver [10] and adrenal gland [7], prevented LAN-induced changes in gene expression.

Here, we demonstrated that early LAN increased glucose levels without an accompanying increase in insulin response. In contrast, late LAN increased the insulin response with only small effects on the glucose response. These data suggest that LAN may affect glucose metabolism by several parallel mechanisms. LAN may have stimulated hepatic glucose production, which would explain the hyperglycaemia and is congruent with the reported upregulation of Pepck in the liver by LAN [10]. Stimulation of glucose production can lead to hyperglycaemia if the insulin response is inadequate. The discrepancy between the adequate insulin response at ZT21 and inadequate response at ZT15 indicates that the observed glucose intolerance at ZT15 is probably due to reduced beta cell sensitivity or an inhibition of insulin release. The inhibitory effect on insulin release seems to be reflected by the reduced baseline insulin concentration at ZT15 and ZT21. Such an inhibitory effect is in line with the previously reported lightinduced reduction of glucose-stimulated insulin secretion [21] and the light-induced decreased parasympathetic and increased sympathetic input to the pancreas [20]. Moreover, in a recent study we found increased fasting and postprandial glucose levels as well as increased sympathetic activity in individuals with type 2 diabetes during early morning exposure to bright light [22].

Glucose intolerance could also have been caused by impaired insulin-independent and/or insulin-dependent glucose uptake by metabolic tissues. Little is known about the role of the ANS in glucose uptake, although it has been suggested that insulin-independent glucose uptake in adipose tissues and skeletal muscle depends on sympathetic innervation [23-26]. Whether light exposure stimulates sympathetic or parasympathetic innervation to muscles in a similar way as to the liver and pancreas $[19,20]$ has not been studied, and whether this would result in an increase or decrease in glucose uptake remains to be determined. However, the IVITT experiments showed that LAN did not reduce whole-body insulin sensitivity.

The discrepancy between insulin responses at the beginning and end of the dark period suggests that the SCN may modulate the light signal towards the pancreas in a timedependent manner. Indeed, many other SCN-mediated effects of light have been shown to differ across the day [10,27], and tracing studies have shown neural connections between the SCN and pancreas [28]. Hypothetically, light-induced SCN stimulation may also affect muscle function in glucose homeostasis, as it was reported that a daily rhythm exists in glucose uptake by muscle tissue in vitro [29], and disruption of the intrinsic muscle clock leads to the disturbance of glucose metabolism [30, 31].

One of the best-studied effects of LAN is the suppression of nocturnal melatonin release by the pineal gland. Experimental $[32,33]$ and genetic studies [34-36] have associated dysfunction of the melatonin system with glucose metabolism. Green and blue light exposure have both been shown to suppress melatonin secretion [37, 38]. However, the distinct glucose responses to LAN with blue and green light in this study make it unlikely that melatonin suppression is a major contributor to the observed glucose intolerance.

Melanopsin, the photosensitive pigment in ipRGCs, is most sensitive to short-wavelength light $(\sim \lambda 450 \mathrm{~nm}$, the blue 
a

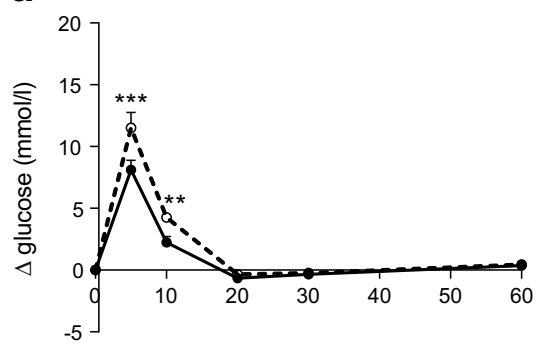

e

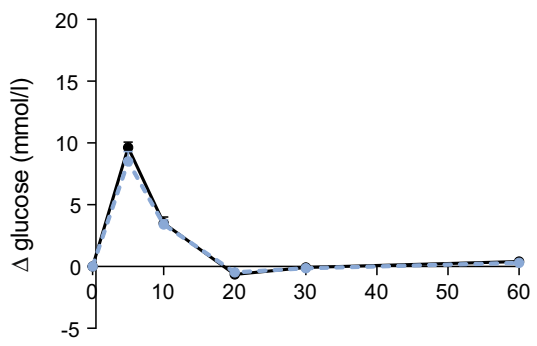

i

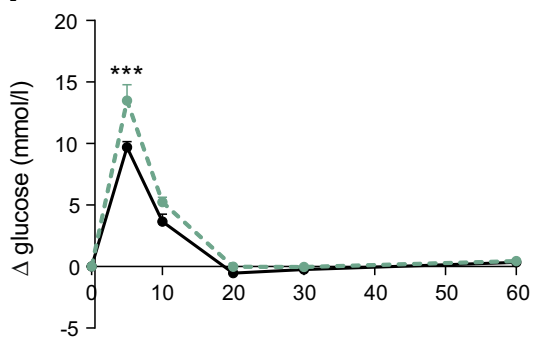

m

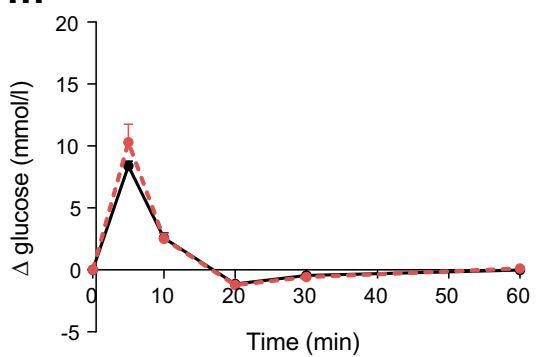

b

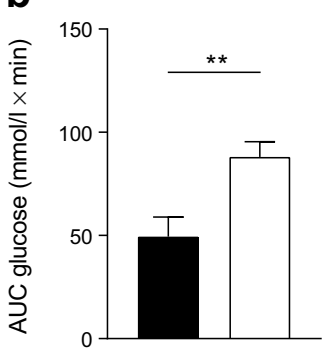

f

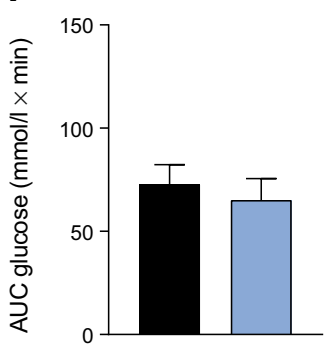

j

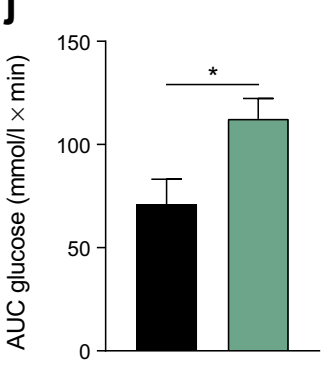

n

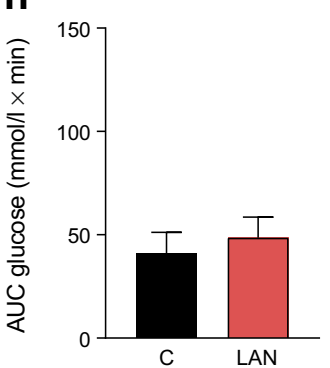

C

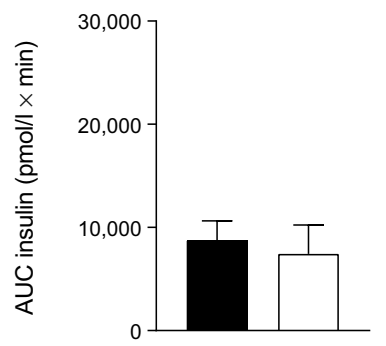

g

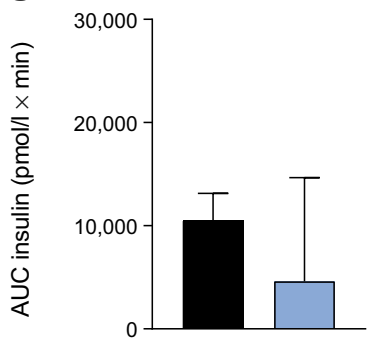

k

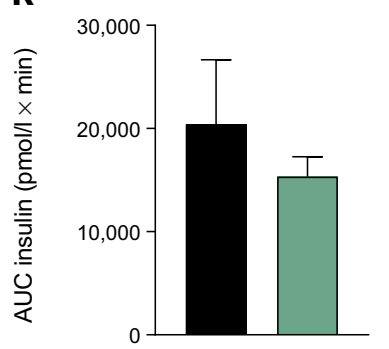

0

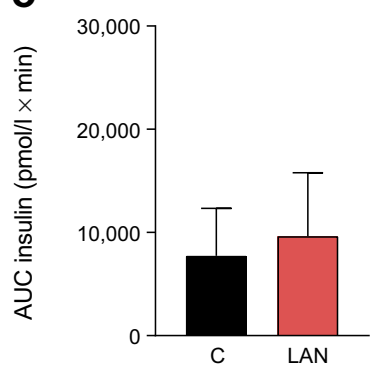

d

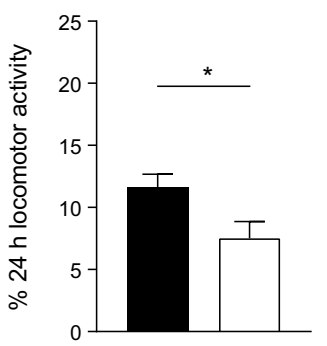

h

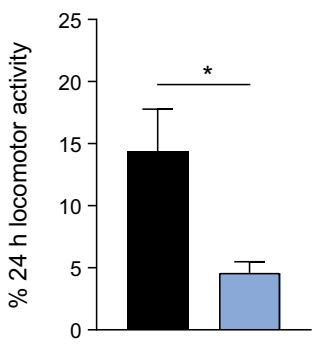

I

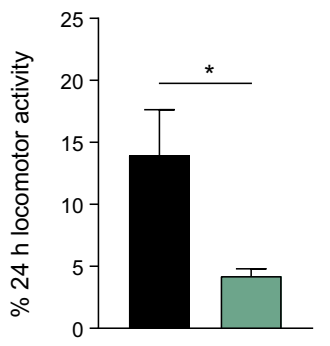

p

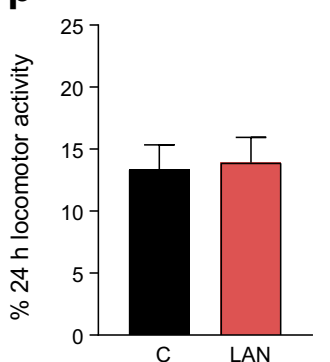

Fig. 5 The effects of LAN on glucose tolerance and locomotor activity depend on wavelength. Two hours of white LAN increased glucose concentrations $(\mathbf{a}, \mathbf{b})$, did not induce changes in the insulin response (c) and decreased total locomotor activity (d). Blue light did not affect glucose concentrations (e, f) or the insulin response (g), but decreased total locomotor activity (h). Green light exposure increased glucose concentrations $(\mathbf{i}, \mathbf{j})$, did not affect the insulin response (k) and decreased total locomotor activity (I). Red light showed no effects on glucose concentration $(\mathbf{m}, \mathbf{n})$, the insulin response (o) or total locomotor activity (p). Control (C), solid lines, black columns and symbols; LAN, dashed lines, white/coloured columns and symbols. $* p<0.05, * * p<0.01, * * * p<0.001$

middle (M-) and long (L-)cones, with M-cones being specifically sensitive to the green spectrum of light $(\lambda 520 \mathrm{~nm})$. Data are limited on the physiological effects of green light and the role of M-cones, besides the suppression of melatonin secretion $[37,38]$ and involvement in circadian clock entrainment [41]. A recent study in mice [40] described distinct melanopsin-dependent effects of blue and green light on sleep, 
a

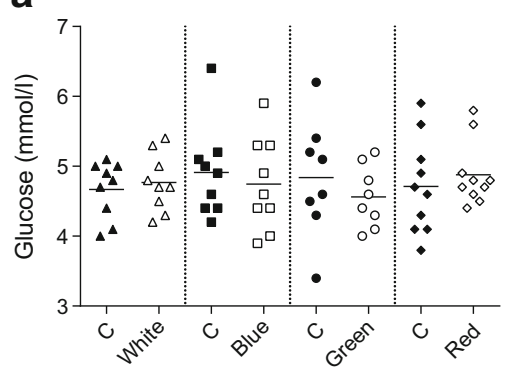

b

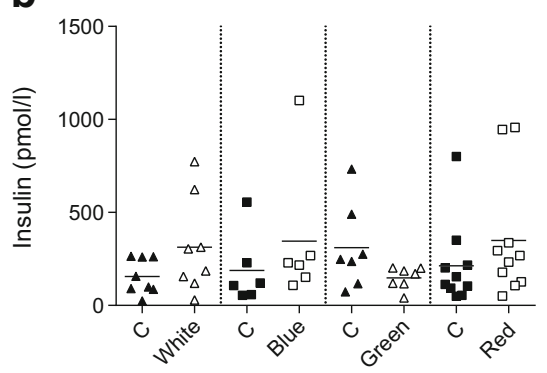

C

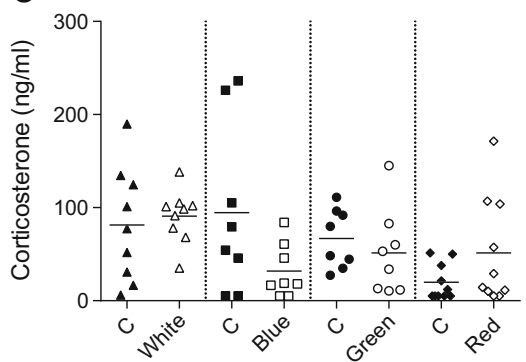

Fig. 6 Baseline concentrations of glucose, insulin and corticosterone are unaffected by LAN at different wavelengths. No significant differences were observed in baseline glucose (a), insulin (b) and corticosterone (c) concentrations between control (black symbols) and LAN conditions (white symbols)

arousal and corticosterone release. The authors proposed that blue light stimulates M1-type ipRGCs projecting to the SCN and thereby acts on arousal and the ANS, whereas green light stimulates a different M-type of ipRGC that projects to the ventrolateral preoptic area and affects sleep. In line with this model, the effects of light on glucose metabolism in our study may be exerted via a non-M1 ipRGC subtype probably projecting preferentially to non-SCN target areas. Indeed, light-evoked effects on heart rate variability [42] and adrenal function [43] were also shown to be independent of ipRGCs innervating the SCN. Nocturnal rodents lack L-cones $\left(\lambda_{\max } 630 \mathrm{~nm}\right)$ and therefore do not show circadian, behavioural or metabolic effects in response to red light $[37,38,44]$. Our study is congruent with this as low illuminance $(5 \mathrm{~lx})$ and red light, main stimulators for L-cones, did not induce glucose intolerance. Furthermore, it is unlikely that S-cones play a significant role as none of our light sources stimulated these photoreceptors (Table 1). In addition to M-cones, rod photoreceptors also respond to green light and, therefore, a mediating role of rods in the effects of light on glucose tolerance cannot be excluded. However, the blue and green LEDs used in our study cause near-equal rod stimulation, whereas $\mathrm{M}$-cone stimulation is higher with green light compared with blue light; therefore, we argue that M-cones are more important than rods in mediating the effects of LAN on glucose metabolism.

The inhibitory effects of LAN on behavioural activity in nocturnal animals are well-known and often reported as masking [45]. Inhibition of locomotor activity may result in reduced glucose uptake by muscle tissue; hypothetically explaining the observed glucose intolerance in this study. However, in view of the distinct responses of locomotion and glucose tolerance to blue light and low intensity white light, it is unlikely that the observed effects on glucose metabolism are mediated via this pathway.

Due to the widespread availability of artificial light, humans and animals around the world are increasingly exposed to LAN and knowledge on the metabolic effects of LAN is thus crucial. Disturbance of the circadian organised physiology by LAN is thought to lead to adverse metabolic
Table 4 Statistical analyses of effects of light on glucose, insulin and corticosterone responses during glucose tolerance tests in experiment 3

\begin{tabular}{llllll}
\hline Wavelength & Variable & $p$ for treatment & $p$ for time & $p$ for interaction & $p$ for AUC \\
\hline \multirow{2}{*}{ White } & Glucose & 0.002 & $<0.001$ & 0.018 & 0.009 \\
& Insulin & 0.616 & $<0.001$ & 0.462 & 0.611 \\
& Corticosterone & 0.041 & $<0.001$ & 0.114 & 0.008 \\
Blue & Glucose & 0.459 & $<0.001$ & 0.154 & 0.602 \\
& Insulin & 0.609 & $<0.001$ & 0.181 & 0.587 \\
& Corticosterone & 0.102 & 0.275 & 0.308 & 0.162 \\
\multirow{3}{*}{ Green } & Glucose & 0.021 & $<0.001$ & 0.001 & 0.027 \\
& Insulin & 0.428 & $<0.001$ & 0.810 & 0.426 \\
& Corticosterone & 0.760 & 0.001 & 0.787 & 0.523 \\
\multirow{2}{*}{ Red } & Glucose & 0.421 & $<0.001$ & 0.172 & 0.603 \\
& Insulin & 0.375 & $<0.001$ & 0.003 & 0.766 \\
& Corticosterone & 0.095 & 0.004 & 0.692 & 0.095 \\
\hline
\end{tabular}

Treatment (wavelength), time (sample time) and interaction effects were determined using repeated measures twoway ANOVA

Glucose, insulin and corticosterone responses are reported for the glucose tolerance test in LAN conditions with four different wavelength spectra

The AUC column contains results of paired $t$ test on the net AUC curve 
health conditions in long-term studies [46]. Our study shows that LAN acutely affects the important homeostatic process of glucose tolerance. The effects on glucose and insulin suggest that light acutely affects the body at multiple levels downstream of the retina and hypothalamus. The nocturnality of rats and the diurnality of humans, as well as the differences in retinal sensitivity, should be taken into consideration when attempting to translate the findings in this study from animal to human. Results of three recent human studies (two performed during daytime and one at nighttime) are nicely in line with our current findings in demonstrating the potential of acute light exposure to affect glucose metabolism [22, 47, 48]. Therefore, chronic and acute light exposure now have been demonstrated to affect glucose metabolism in both rodent [4] and human studies [1-3, 22, 47, 48]. Chronic and acute LAN exposure are both representative conditions for human shift workers [46, 49], as well as for the global community exposed to increasing light pollution and the growing use of light-emitting products. Our study increases the knowledge of light effects on physiology, which may contribute to a better understanding of the correlation between LAN, shift work and metabolic disorders including diabetes.

In conclusion, we showed in rats that nocturnal light exposure acutely induced glucose intolerance, possibly via reduced beta cell sensitivity. This effect depended on the time of day, pointing towards an interaction with circadian physiology. The effect of light on glucose tolerance was also dependent on intensity and wavelength, suggesting a mediating role of non-M1 ipRGCs and M-cone photoreceptors. Our study warrants attention for the potentially hazardous actions of light on health in order to control the regulation of design and use of light-emitting products, along with advice for and protection of frequently exposed individuals.

Data availability The data that support the findings of this study are available from the corresponding author upon reasonable request.

Funding This study is funded by STW grant OnTime (project 12195). DJS is supported by ZonMW (AGIKO 92003592).

Duality of interest The authors declare that there is no duality of interest associated with this manuscript.

Author contributions All authors provided substantial contributions to conception and design (ALO, DJS, AK), acquisition of data (ALO, RDJ, $\mathrm{EFo}$ ), or analysis and interpretation of data (ALO, EFl, AK). Authors contributed to drafting the article (ALO, AK) or revising it (ALO, DJS, RDJ, EFo, EFl) critically for important intellectual content. All authors approved the final version of this manuscript. AK is the guarantor of this work.

Open Access This article is distributed under the terms of the Creative Commons Attribution 4.0 International License (http:// creativecommons.org/licenses/by/4.0/), which permits unrestricted use, distribution, and reproduction in any medium, provided you give appropriate credit to the original author(s) and the source, provide a link to the Creative Commons license, and indicate if changes were made.

\section{References}

1. McFadden E, Jones ME, Schoemaker MJ, Ashworth A, Swerdlow $\mathrm{AJ}$ (2014) The relationship between obesity and exposure to light at night: cross-sectional analyses of over 100,000 women in the Breakthrough Generations Study. Am J Epidemiol 180:245-250

2. Obayashi K, Saeki K, Iwamoto J et al (2013) Exposure to light at night, nocturnal urinary melatonin excretion, and obesity/ dyslipidemia in the elderly: a cross-sectional analysis of the HEIJO-KYO study. J Clin Endocrinol Metab 98:337-344

3. Obayashi K, Saeki K, Iwamoto J, Ikada Y, Kurumatani N (2014) Independent associations of exposure to evening light and nocturnal urinary melatonin excretion with diabetes in the elderly. Chronobiol Int 31:394-400

4. Fonken LK, Workman JL, Walton JC et al (2010) Light at night increases body mass by shifting the time of food intake. Proc Natl Acad Sci U S A 107:18,664-18,669

5. Coomans CP, van den Berg SA, Houben T et al (2013) Detrimental effects of constant light exposure and high-fat diet on circadian energy metabolism and insulin sensitivity. FASEB J 27:1721-1732

6. Stenvers DJ, van Dorp R, Foppen E et al (2016) Dim light at night disturbs the daily sleep-wake cycle in the rat. Sci Rep 6:35662

7. Ishida A, Mutoh T, Ueyama T et al (2005) Light activates the adrenal gland: timing of gene expression and glucocorticoid release. Cell Metab 2:297-307

8. Kalsbeek A, Cutrera RA, van Heerikhuize JJ, van der Vliet J, Buijs RM (1999) GABA release from suprachiasmatic nucleus terminals is necessary for the light-induced inhibition of nocturnal melatonin release in the rat. Neuroscience 91:453-461

9. Best JD, Maywood ES, Smith KL, Hastings MH (1999) Rapid resetting of the mammalian circadian clock. J Neurosci 19:828-835

10. Cailotto C, Lei J, van der Vliet J et al (2009) Effects of nocturnal light on (clock) gene expression in peripheral organs: a role for the autonomic innervation of the liver. PLoS One 4:e5650

11. La Fleur SE, Kalsbeek A, Wortel J, Buijs RM (1999) A suprachiasmatic nucleus generated rhythm in basal glucose concentrations. J Neuroendocrinol 11:643-652

12. La Fleur SE, Kalsbeek A, Wortel J, Fekkes ML, Buijs RM (2001) A daily rhythm in glucose tolerance: a role for the suprachiasmatic nucleus. Diabetes 50:1237-1243

13. Cailotto C, la Fleur SE, van Heijningen C et al (2005) The suprachiasmatic nucleus controls the daily variation of plasma glucose via the autonomic output to the liver: are the clock genes involved? Eur J Neurosci 22:2531-2540

14. Provencio I, Rodriguez IR, Jiang G, Hayes WP, Moreira EF, Rollag MD (2000) A novel human opsin in the inner retina. J Neurosci 20: 600-605

15. Hattar S, Lucas RJ, Mrosovsky N et al (2003) Melanopsin and rodcone photoreceptive systems account for all major accessory visual functions in mice. Nature 424:76-81

16. Dollet A, Albrecht U, Cooper HM, Dkhissi-Benyahya O (2010) Cones are required for normal temporal responses to light of phase shifts and clock gene expression. Chronobiol Int 27:768-781

17. Lucas RJ, Lall GS, Allen AE, Brown TM (2012) How rod, cone, and melanopsin photoreceptors come together to enlighten the mammalian circadian clock. Prog Brain Res 199:1-18

18. Lucas RJ, Peirson SN, Berson DM et al (2014) Measuring and using light in the melanopsin age. Trends Neurosci 37:1-9 
19. Niijima A, Nagai K, Nagai N, Akagawa H (1993) Effects of light stimulation on the activity of the autonomic nerves in anesthetized rats. Physiol Behav 54:555-561

20. Niijima A, Nagai K, Nagai N, Nakagawa H (1992) Light enhances sympathetic and suppresses vagal outflows and lesions including the suprachiasmatic nucleus eliminate these changes in rats. J Auton Nerv Syst 40:155-160

21. Qian J, Block GD, Colwell CS, Matveyenko AV (2013) Consequences of exposure to light at night on the pancreatic islet circadian clock and function in rats. Diabetes 62:3469-3478

22. Versteeg RI, Stenvers DJ, Visintainer D, et al (2017) Acute effects of morning light on plasma glucose and triglycerides in healthy men and men with type 2 diabetes. J Biol Rhythms. doi:10.1177/ 0748730417693480

23. Haque MS, Minokoshi Y, Hamai M, Iwai M, Horiuchi M, Shimazu $\mathrm{T}$ (1999) Role of the sympathetic nervous system and insulin in enhancing glucose uptake in peripheral tissues after intrahypothalamic injection of leptin in rats. Diabetes 48:17061712

24. Minokoshi Y, Okano Y, Shimazu T (1994) Regulatory mechanism of the ventromedial hypothalamus in enhancing glucose uptake in skeletal muscles. Brain Res 649:343-347

25. Shimazu T, Sudo M, Minokoshi Y, Takahashi A (1991) Role of the hypothalamus in insulin-independent glucose uptake in peripheral tissues. Brain Res Bull 27:501-504

26. Sudo M, Minokoshi Y, Shimazu T (1991) Ventromedial hypothalamic stimulation enhances peripheral glucose uptake in anesthetized rats. Am J Phys 261:E298-E303

27. Buijs RM, Wortel J, van Heerikhuize JJ et al (1999) Anatomical and functional demonstration of a multisynaptic suprachiasmatic nucleus adrenal (cortex) pathway. Eur J Neurosci 11:1535-1544

28. Buijs RM, Chun SJ, Niijima A, Romijn HJ, Nagai K (2001) Parasympathetic and sympathetic control of the pancreas: a role for the suprachiasmatic nucleus and other hypothalamic centres that are involved in the regulation of food intake. J Comp Neurol 431: 405-423

29. Feneberg R, Lemmer B (2004) Circadian rhythm of glucose uptake in cultures of skeletal muscle cells and adipocytes in Wistar-Kyoto, Wistar, Goto-Kakizaki, and spontaneously hypertensive rats. Chronobiol Int 21:521-538

30. Dyar KA, Ciciliot S, Wright LE et al (2014) Muscle insulin sensitivity and glucose metabolism are controlled by the intrinsic muscle clock. Mol Metab 3:29-41

31. Harfmann BD, Schroder EA, Kachman MT, Hodge BA, Zhang X, Esser KA (2016) Muscle-specific loss of Bmall leads to disrupted tissue glucose metabolism and systemic glucose homeostasis. Skelet Muscle 6:12

32. La Fleur SE, Kalsbeek A, Wortel J, van der Vliet J, Buijs RM (2001) Role for the pineal and melatonin in glucose homeostasis: pinealectomy increases night-time glucose concentrations. J Neuroendocrinol 13:1025-1032

33. Picinato MC, Haber EP, Carpinelli AR, Cipolla-Neto J (2002) Daily rhythm of glucose-induced insulin secretion by isolated islets from intact and pinealectomized rat. J Pineal Res 33:172-177
34. Bouatia-Naji N, Bonnefond A, Cavalcanti-Proenca C et al (2009) A variant near MTNRIB is associated with increased fasting plasma glucose levels and type 2 diabetes risk. Nat Genet 41:89-94

35. Lyssenko V, Nagorny CL, Erdos MR et al (2009) Common variant in MTNR1B associated with increased risk of type 2 diabetes and impaired early insulin secretion. Nat Genet 41:82-88

36. Sparso T, Bonnefond A, Andersson E et al (2009) G-allele of intronic rs 10830963 in MTNR1B confers increased risk of impaired fasting glycemia and type 2 diabetes through an impaired glucosestimulated insulin release: studies involving 19,605 Europeans. Diabetes 58:1450-1456

37. Brainard GC, Hanifin JP, Greeson JM et al (2001) Action spectrum for melatonin regulation in humans: evidence for a novel circadian photoreceptor. J Neurosci 21:6405-6412

38. Wright HR, Lack LC, Kennaway DJ (2004) Differential effects of light wavelength in phase advancing the melatonin rhythm. J Pineal Res 36:140-144

39. Lockley SW, Brainard GC, Czeisler CA (2003) High sensitivity of the human circadian melatonin rhythm to resetting by short wavelength light. J Clin Endocrinol Metab 88:4502-4505

40. Pilorz V, Tam SK, Hughes S et al (2016) Melanopsin regulates both sleep-promoting and arousal-promoting responses to light. PLoS Biol 14:e1002482

41. Dkhissi-Benyahya O, Gronfier C, De Vanssay W, Flamant F, Cooper HM (2007) Modeling the role of mid-wavelength cones in circadian responses to light. Neuron 53:677-687

42. Thompson S, Lupi D, Hankins MW, Peirson SN, Foster RG (2008) The effects of rod and cone loss on the photic regulation of locomotor activity and heart rate. Eur J Neurosci 28:724-729

43. Kiessling S, Sollars PJ, Pickard GE (2014) Light stimulates the mouse adrenal through a retinohypothalamic pathway independent of an effect on the clock in the suprachiasmatic nucleus. PLoS One 9:e92959

44. Bedrosian TA, Vaughn CA, Galan A, Daye G, Weil ZM, Nelson RJ (2013) Nocturnal light exposure impairs affective responses in a wavelength-dependent manner. J Neurosci 33:13,081-13,087

45. Mrosovsky N (1999) Masking: history, definitions, and measurement. Chronobiol Int 16:415-429

46. Wyse CA, Selman C, Page MM, Coogan AN, Hazlerigg DG (2011) Circadian desynchrony and metabolic dysfunction; did light pollution make us fat? Med Hypotheses 77:1139-1144

47. Cheung IN, Zee PC, Shalman D, Malkani RG, Kang J, Reid KJ (2016) Morning and evening blue-enriched light exposure alters metabolic function in normal weight adults. PLoS One 11: e0155601

48. Albreiki MS, Middleton B, Hampton SM (2017) A single night light exposure acutely alters hormonal and metabolic responses in healthy participants. Endocr Connect 6:100-110

49. Opperhuizen AL, van Kerkhof LW, Proper KI, Rodenburg W, Kalsbeek A (2015) Rodent models to study the metabolic effects of shiftwork in humans. Front Pharmacol 6:50 